\title{
Thresholds for Sterol-Limited Growth of Daphnia magna: A Comparative Approach Using 10 Different Sterols
}

\author{
Dominik Martin-Creuzburg • Sarah Oexle • \\ Alexander Wacker
}

\begin{abstract}
Arthropods are incapable of synthesizing sterols de novo and thus require a dietary source to cover their physiological demands. The most prominent sterol in animal tissues is cholesterol, which is an indispensable structural component of cell membranes and serves as precursor for steroid hormones. Instead of cholesterol, plants and algae contain a variety of different phytosterols. Consequently, herbivorous arthropods have to metabolize dietary phytosterols to cholesterol to meet their requirements for growth and reproduction. Here, we investigated sterol-limited growth responses of the freshwater herbivore Daphnia magna by supplementing a sterol-free diet with increasing amounts of 10 different phytosterols and comparing thresholds for sterol-limited growth. In addition, we analyzed the sterol composition of $D$. magna to explore sterol metabolic constraints and bioconversion capacities. We show that dietary phytosterols strongly differ in their potential to support somatic growth of D. magna. The dietary threshold concentrations obtained by supplementing the different sterols cover a wide range (3.5-34.4 $\mu \mathrm{g} \mathrm{mg} \mathrm{C}^{1}$ )
\end{abstract}

D. Martin Creuzburg $(\bowtie) \cdot$ S. Oexle

Limnological Institute, University of Konstanz,

Mainaustrasse 252, 78464 Konstanz, Germany

e mail: Dominik.Martin Creuzburg@uni konstanz.de

\section{A. Wacker}

Institute for Biochemistry and Biology, Ecology and Ecosystem Modelling, University of Potsdam,

Maulbeerallee 2, 14469 Potsdam, Germany

Present Address:

S. Oexle

Laboratory of Aquatic Ecology, Evolution and Conservation, Catholic University of Leuven,

Charles Deberiotstraat 32, box 2439, 3000 Leuven, Belgium and encompass the one for cholesterol $\left(8.9 \mu \mathrm{g} \mathrm{mg} \mathrm{C}^{1}\right)$, indicating that certain phytosterols are more efficient in supporting somatic growth than cholesterol (e.g., fucosterol, brassicasterol) while others are less efficient (e.g., dihydrocholesterol, lathosterol). The dietary sterol concentration gradients revealed that the poor quality of particular sterols can be alleviated partially by increasing dietary concentrations, and that qualitative differences among sterols are most pronounced at low to moderate dietary concentrations. We infer that the dietary sterol composition has to be considered in zooplankton nutritional ecology to accurately assess potential sterol limitations under field conditions.

Keywords Cholesterol · Daphnia $\cdot$ Food quality $\cdot$ Nutrition . Phytosterols $\cdot$ Sterols

\section{Introduction}

Sterols are indispensable structural components of eukaryotic cell membranes; they are involved in the modulation of important membrane properties, such as permeability and fluidity, and they interact with various membrane proteins (Haines 2001; Ohvo-Rekilä et al. 2002). Moreover, sterols serve as precursors for steroid hormones, such as the molt-inducing ecdysteroids in arthropods (Grieneisen 1994), and are required for developmental patterning of embryonic structures (Porter et al. 1996). Generally, it is accepted that arthropods require dietary sterols to satisfy their physiological demands (Behmer and Nes 2003; Goad 1981; Hassett 2004; Martin-Creuzburg and von Elert 2009). Moreover, nematodes, rotifers, and certain bivalves are either incapable of synthesizing sterols de novo or at least have limited capacities to synthesize sterols from low molecular weight precursors (Basen et al. 2012; Entchev and Kurzchalia 2005; Wacker and MartinCreuzburg 2012). Thus, sterol auxotrophy appears to be 
widespread among invertebrates. In contrast to animals, which predominantly contain cholesterol as their main body sterol (Goad 1981), plants and especially algae contain a great diversity of phytosterols and little to no cholesterol (Behmer et al. 2011; Volkman 2003). These phytosterols typically differ from cholesterol in the number and position of double bonds in the tetracyclic sterol nucleus or in the side chain or by having additional substituents, such as methyl or ethyl groups at C-24 in the side chain (Moreau et al. 2002). Herbivorous arthropods rely on a dietary source of sterols, and typically produce cholesterol by converting the phytosterols in their diet (Ikekawa et al. 1993; Svoboda 1999). Work on insects and crustaceans has shown that phytosterols can differ substantially in their potential to support growth and reproduction (Behmer and Nes 2003; Carvalho et al. 2010; MartinCreuzburg and von Elert 2009). Although it has been recognized that many animals rely on an adequate dietary supply of sterols and other essential biochemicals, dietary threshold concentrations at which these compounds become limiting have not been adequately studied, in particular with regard to non-insect arthropods.

Here, we investigated the sterol requirements of Daphnia magna, an important component of freshwater food webs and well-established model organism in ecology, ecotoxicology, and evolutionary research. It has been shown that a dietary sterol deficiency has serious consequences for growth, reproduction and survival of Daphnia species (Martin-Creuzburg et al. 2005b, 2008, 2011). Dietary thresholds for sterol-limited growth have been determined only for cholesterol (Martin-Creuzburg et al. 2009, 2010; Sperfeld and Wacker 2009). The use of cholesterol to assess sterol requirements is justified by its physiological relevance; cholesterol is the predominant body sterol in Daphnia as in most other animals. However, this approach neglects the fact that Daphnia primarily feed on algae, which generally do not contain cholesterol but different phytosterols (Volkman 2003). It has been shown already that dietary phytosterols can differ substantially in their potential to support growth and reproduction of Daphnia galeata (Martin-Creuzburg and von Elert 2004). However, quantitative differences in phytosterol requirements have not yet been explored. Here, we offered various concentrations of ten different sterols as dietary supplements to a sterol-free food source provided to juvenile D. magna. Somatic growth rates in response to increasing dietary sterol concentrations and thresholds for sterol-limited growth were evaluated. We hypothesized that the sterollimited growth responses, and thus the dietary threshold concentrations for sterol-limited growth are affected by structural differences among sterols. In addition, the concentrations of individual sterols in D. magna tissues were determined to obtain information on sterol metabolic constraints and bioconversion capacities.

\section{Methods and Materials}

Cultivation of Organisms and Preparation of Food Suspensions Stock cultures of a clone of Daphnia magna Straus, 1820, originally isolated from 'Großer Binnensee', Germany (Lampert 1991), were raised in filtered lake water $(0.2 \mu \mathrm{m}$ pore-sized membrane filter) containing saturating concentrations ( $2 \mathrm{mg} \mathrm{C}^{1}$ ) of the green alga Scenedesmus obliquus (Culture collection of algae, University of Göttingen, Germany, SAG 276-3a). S. obliquus was grown at $20{ }^{\circ} \mathrm{C}$ in batch cultures as described previously (MartinCreuzburg et al. 2008). For the growth experiment, the cyanobacterium Synechococcus elongatus (SAG 89.79) was cultivated semi-continuously at a dilution rate of $0.25 \mathrm{~d}^{1} ; S$. elongatus was chosen because it is non-toxic, phosphorus-rich, and well-assimilated by Daphnia but lacks sterols (Martin-Creuzburg et al. 2008, and references therein). S. elongatus was harvested daily, and food suspensions were prepared by centrifugation and resuspension in fresh medium. Carbon concentrations of the cyanobacterial food suspensions were estimated from photometric light extinctions (800 nm) using a previously established carbonextinction regression.

Growth Experiment Second-clutch juveniles of D. magna born within $12 \mathrm{~h}$ were used for the growth experiment. The experiment was carried out at $20^{\circ} \mathrm{C}$ with a 16:8 $\mathrm{h} \mathrm{L}: \mathrm{D}$ cycle in glass beakers filled with 0.21 of filtered lake water $(<0.2 \mu \mathrm{m})$. Animals were reared on saturating concentrations of S. elongatus (2 $\mathrm{mg} \mathrm{C}^{1}$ ). Sterol supplementation was achieved by adding increasing amounts of sterol-containing liposomes to the experimental beakers. In this way, seven sterol concentrations were provided $(0,2.1,4.1,8.2,16.5$, 33.0, and $41.2 \mu \mathrm{g} \mathrm{mg} \mathrm{C}{ }^{1}$ ). S. elongatus is deficient not only in sterols but also in essential polyunsaturated fatty acids (PUFA; Martin-Creuzburg et al. 2008). Thus, in addition to sterol-containing liposomes, each beaker was supplemented with $20 \mu \mathrm{l}$ of eicosapentaenoic acid (EPA)-containing liposomes, corresponding to an EPA concentration of $(30 \mu \mathrm{g} \mathrm{mg}$ $\mathrm{C}^{1}$ ), to release the animals from a simultaneous PUFA limitation (Martin-Creuzburg et al. 2009; Sperfeld et al. 2012). To account for the increasing carbon and phosphorus (constituents of phospholipids) supply via supplementary liposomes, unloaded (i.e., sterol- and EPA-free) control liposomes were added so that each beaker was provided in total with $120 \mu$ of liposomes. Each treatment consisted of three replicates with six juvenile D. magna per beaker. During the experiment, daphnids were transferred daily into new beakers with freshly prepared food suspensions. At the end of the experiment on day 5 , daphnids were stored at $-80{ }^{\circ} \mathrm{C}$, freeze-dried, and weighed (Mettler Toledo XP2U; $\pm 0.1 \mu \mathrm{g}$ ). Juvenile somatic growth rates (g) were determined as the increase in dry mass from the beginning of the experiment 
$\left(\mathrm{M}_{0}\right)$ today $5\left(\mathrm{M}_{\mathrm{t}}\right)$ with time (t) expressed as age in days:

$g=\frac{\operatorname{In} M_{\mathrm{t}}-\operatorname{In} M_{0}}{t}$

Liposome Preparation Liposome stock suspensions were prepared by dissolving $3 \mathrm{mg}$ 1-palmitoyl-2-oleoylphosphatidylglycerol (POPG) and $7 \mathrm{mg}$ 1-palmitoyl-2oleoylphosphatidylcholin (POPC; Lipoid, Germany) in an aliquot of ethanol. Sterol-containing liposomes were prepared by adding $3.33 \mathrm{mg}$ of sterol stock solutions dissolved in ethanol. The resulting solutions were dried using a rotary evaporator and dissolved in $10 \mathrm{ml}$ buffer $\left(20 \mathrm{mmol} 1^{1} \mathrm{Na}\right.$ phosphate, $150 \mathrm{mmol} 1{ }^{1} \mathrm{NaCl}, \mathrm{pH}$ 7.0). Subsequently, suspensions were sonicated in an ultrasonic bath. Excess free sterols were removed by washing the liposomes in fresh buffer using an ultracentrifuge $\left(150,000 \mathrm{~g}, 90 \mathrm{~min}, 4^{\circ} \mathrm{C}\right)$. Prior to the addition of liposomes to the experimental beakers, liposome stock suspensions were sonicated again $(2 \mathrm{~min})$. All sterols were obtained from Sigma Aldrich (St. Louis, MO, USA).

Chemical Analysis The sterol composition of D. magna reared at high dietary sterol concentrations $\left(33 \mu \mathrm{g} \mathrm{mg} \mathrm{C}{ }^{1}\right)$ was determined. For the analysis, freeze-dried and weighed daphnids were deposited in dichloromethane/methanol (2:1, $v / v)$, vigorously sonicated, and stored overnight at $-20{ }^{\circ} \mathrm{C}$. On the next day, $5 \alpha$-cholestane was added as internal standard (1.000-3.000 ng per sample, depending on the expected sterol concentrations), and total lipids were extracted using two washes with dichloromethane/methanol (2:1, $v / v)$. The pooled cell-free lipid extracts were evaporated to dryness under $\mathrm{N}_{2}$-atmosphere and saponified with methanolic $\mathrm{KOH}\left(0.2 \mathrm{~mol} 1{ }^{1}, 70{ }^{\circ} \mathrm{C}, 1 \mathrm{~h}\right)$. Subsequently, the neutral lipids were partitioned into iso-hexane:diethylether (9:1, $\mathrm{v}: \mathrm{v})$, evaporated to dryness under $\mathrm{N}_{2}$, and resuspended in iso-hexane (10-30 $\mathrm{\mu l}$, depending on the amount of $5 \alpha$ cholestane that was previously added). Sterols were analyzed and quantified by gas chromatography on an HP 6,890 GC equipped with a flame ionization detector (FID) and an HP-5 (Agilent, $30 \mathrm{~m} \times 0.25 \mathrm{~mm} \mathrm{ID} \times 0.25 \mathrm{~mm}$ film) capillary column. The following configurations were used: oven, $150{ }^{\circ} \mathrm{C}(1 \mathrm{~min})$ to $280{ }^{\circ} \mathrm{C}$ at $15{ }^{\circ} \mathrm{C}$ min ${ }^{1}$, then to $320{ }^{\circ} \mathrm{C}$ at $2{ }^{\circ} \mathrm{C}$ min ${ }^{1}$; carrier gas, helium (flow, $1.5 \mathrm{ml} \mathrm{min}{ }^{1}$; velocity, $38 \mathrm{~cm} \mathrm{~s}^{1}$ ); detector, FID $350{ }^{\circ} \mathrm{C}$; injector, $350{ }^{\circ} \mathrm{C}$ (total run time 30 min sample ${ }^{1}$ ). The injection volume was $1 \mu \mathrm{l}$ (equivalent to $100 \mathrm{ng} 5 \alpha$ cholestane). Sterols were quantified by comparison to $5 \alpha-$ cholestane using multipoint calibration curves generated for each of the supplementary sterols; the few detected in D. magna that were not commercially available were quantified as cholesterol-equivalents using the cholesterol calibration curve. Sterols were identified by their retention times and their mass spectra, which were recorded with a gas chromatograph-mass spectrometer (GC-MS) (Agilent Technologies, 5975C inert MSD) equipped with a fused-silica capillary column (DB-5MS, Agilent; GC-MS configurations as described for FID). Sterols were analyzed both in their free form and as their trimethylsilyl derivatives, which were prepared by incubating $20 \mu \mathrm{l}$ of the iso-hexane sterol extracts with $10 \mu l$ of N,O-bis (trimethylsilyl) trifluoroacetamide (BSTFA) including $1 \%$ trimethylchlorosilane (TMCS) for $1 \mathrm{~h}$ at room temperature. Spectra were recorded between 50 and 600 Dalton in the electron impact (EI) ionization mode. The limit for quantitation of sterols was $20 \mathrm{ng}$. The absolute amount of each sterol was related to the number/dry mass of the animals. Sterols in the food/liposome suspensions were related to the particulate organic carbon content, which was determined using an elemental analyser (EuroEA3000, HEKAtech GmbH, Germany).

Data Analysis Sterol-limited growth responses and dietary thresholds were estimated based on saturation curve fitting (Sperfeld and Wacker 2011) using a modified form of the Von Bertalanffy function (Bertalanffy 1957), which describes somatic growth rates $\left(g, \mathrm{~d}^{1}\right)$ in response to dietary nutrient concentrations ( $c$, in $\mu \mathrm{g} \mathrm{mg} \mathrm{C}{ }^{1}$ ).

$g=g_{0}+\left(g_{\infty}-g_{0}\right) \cdot\left(1-\exp \left(-b^{*} c\right)\right)$

The parameters are: $g_{0}=$ somatic growth rate $\left(\mathrm{d}^{1}\right)$ on unsupplemented $S$. elongatus, $g_{\infty}=$ asymptotic growth rate $\left(\mathrm{d}^{1}\right), b=$ Bertalanffy growth coefficient in $\mathrm{mg} \mathrm{C}\left(\mu \mathrm{g}\right.$ sterol) ${ }^{1}$.

To estimate the uncertainty of each sterol threshold depending on the variability of growth data, we randomly sampled one out of three replicates for each of the original dietary sterol concentration levels and used Eq. 2 to fit through the sampled growth rates using the non-linear least squares method of $\mathrm{R}$ 2.11.1. From the fitted curves, we calculated the sterol concentrations at which maximum growth rates $\left(g_{\infty}\right)$ were reduced by $25 \%$, and we defined these concentrations as thresholds for sterol-limited growth. A growth rate reduction of $25 \%$ was used because the variability in threshold concentrations increases with increasing convergence to $g_{\infty}$, and this may compromise further evaluations (Sperfeld and Wacker 2011). The value for $g_{\infty}$ cannot be used as estimation for growth saturation because saturation curves reach $g_{\infty}$ at infinity (Fig. 1). Thus, we defined a saturation growth level clearly distinct to $g_{\infty}$ on the basis of the maximum growth improvement by dietary sterol supply (Fig. 1). Using this conservative saturation growth level (reduction of $g_{\infty}$ by $25 \%$ ) may result in a reduced sensitivity to detect growth limitations. However, this procedure clearly avoids biased threshold estimations due to neglecting some bootstrapped regression curves that do not intersect with the line of the defined saturation growth level, and allows for an unbiased comparison of sterol thresholds. 

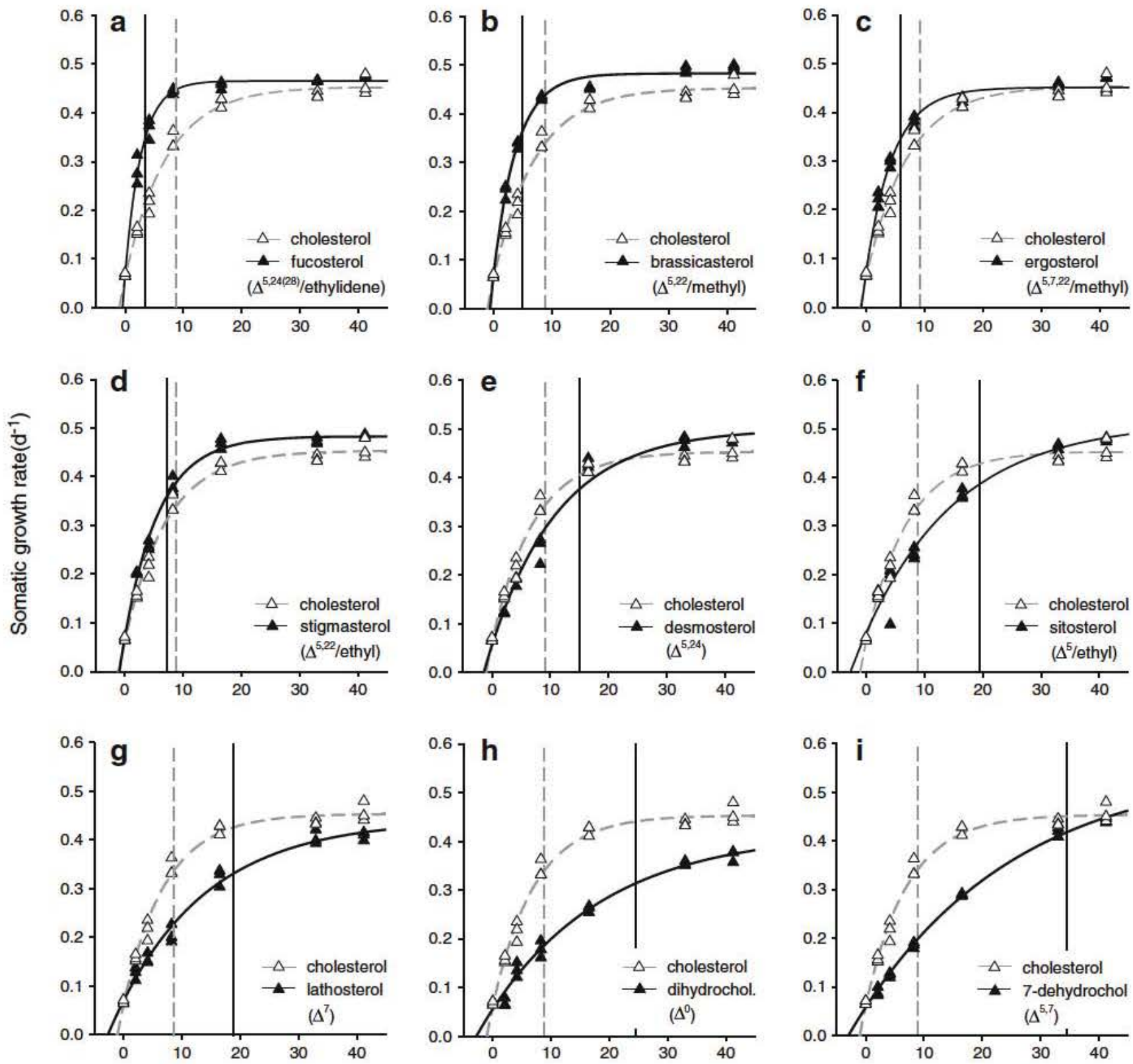

Dietary sterol concentration ( $\mu \mathrm{g} \mathrm{mg} \mathrm{C}^{-1}$ )

Fig. 1 Somatic growth rates of Daphnia magna in response to increasing dietary sterol concentrations. The growth response curves were recorded for cholesterol (open triangles) and nine other sterols (black triangles; a i); the growth response curve for cholesterol is plotted in each graph for

By repeating this procedure 1,000 times, a parameter distribution around a median threshold was generated and used for further statistical comparison of thresholds among treatments.

To statistically evaluate potential differences among the thresholds obtained for the different sterols, we randomly drew the same number of replicates as in the original experiment $(N=3)$ from the distribution of the 1,000 thresholds. This was done for each sterol treatment; the resulting replicates were compared using analysis of variance followed by Tukey's multiple comparis on test. Following the comparison (seven dietary sterol concentrations, $N=3$ each). Vertical lines show the dietary threshold concentrations for sterol limited growth (de fined as a growth rate reduction of $\mathrm{g}_{\infty}$ by $25 \%$ ) for cholesterol (grey dashed lines) and for the other sterols (black solid lines)

recommendation of Bolker (2008), this procedure was repeated 400 times. The proportion of significant tests, i.e., with $P<0.05$ for each comparison, reflected the probability and power to detect significant differences among treatments. We searched for the critical $P$-value necessary to exceed an $80 \%$ proportion of significant tests $(=80 \%$ percentile) to hold for a statistical power of 0.8 (or a type two error of 0.2 , respectively). According to an in $\mathrm{R}$ implemented algorithm based on Piepho (2004), we used these critical $P$-values to identify homogenous groups among treatments. The total 
body sterol content of $D$. magna was analysed using ANOVA. To account for inhomogeneity of variances, the raw data were transformed by taking the 4-th root. Treatment effects were analyzed using Tukey's HSD post hoc test.

\section{Results}

Sterol limited Growth Responses of D. magna The sterols used for supplementation were selected because they are either frequently detected in phytoplankton samples (i.e., fucosterol, brassicasterol, ergosterol, stigmasterol, sitosterol), important in Daphnia physiology (i.e., cholesterol, 7dehydrocholesterol), or represent structural properties (additional double bonds, alkyl substituents) that may help to elucidate metabolic pathways and sterol metabolic constraints (Table 1). Juvenile somatic growth rates (g) of D. magna fed unsupplemented S. elongatus were low, but increased considerably with increasing dietary sterol concentrations (Fig. 1). The sterol-limited growth responses obtained by increasing the availability of different dietary sterols varied in their initial slopes and their maximum obtained growth rates, resulting in different threshold levels at which sterol saturated growth passed into sterol limited growth (defined as a reduction of $\mathrm{g}_{\infty}$ by $25 \%$; Fig. 1). The highest thresholds for sterol-limited growth (indicating large amounts of dietary sterols required) were obtained with lathosterol, dihydrocholesterol, and 7dehydrocholesterol (Figs. 1, 2). At the highest dietary concentration, somatic growth rates obtained with cholesterol or 7dehydrocholesterol supplementation were similar (Fig. 1).

Supplementation with desmosterol or sitosterol also revealed higher thresholds for sterol limited growth than were obtained with cholesterol supplementation. At high dietary concentrations, desmosterol or sitosterol supported slightly higher growth rates than cholesterol, resulting in an intersection of the growth curves (Fig. 1). The growth response curves obtained by supplementation with stigmasterol or cholesterol were rather similar and did not reveal significant differences in dietary threshold concentrations (Fig. 2). The growth curves obtained by supplementation with fucosterol, brassicasterol, or ergosterol revealed significantly lower thresholds for sterol limited growth than the growth curve obtained with cholesterol supplementation (Figs. 1, 2). The dietary threshold concentrations for sterol limited growth ranged between 3.5 (fucosterol) and $34.3 \mu \mathrm{g} \mathrm{mg} \mathrm{C}{ }^{1}$ (7-dehydrocholesterol); the threshold for cholesterol was $8.9 \mu \mathrm{g} \mathrm{mg} \mathrm{C}{ }^{1}$ (Fig. 2).

Sterol Content and Composition of D. magna The total body sterol content of $D$. magna was significantly affected by the dietary sterol supply (ANOVA, $F_{11,23}=215.7, P<0.001$ ). When reared on unsupplemented (i.e., sterol-free) S. elongatus, the total body sterol content did not differ from that of animals subsampled at the start of the experiment (Fig. 3; Tukey's HSD, $P=0.99$ ). The total body sterol content increased considerably upon dietary sterol supplementation. Animals reared on diets containing high concentrations (33 $\mu \mathrm{g} \mathrm{mg} \mathrm{C}^{1}$ ) of dihydrocholesterol or 7-dehydrocholesterol, i.e., the two sterols that had the highest dietary threshold concentrations had the lowest total body sterol content (Fig. 3). Animals reared on diets containing high concentrations of fucosterol, the sterol that had the lowest dietary threshold concentration, had the highest total body sterol content. A regression analysis revealed a weak positive correlation between somatic growth rates at high dietary sterol concentrations (33 $\left.\mu \mathrm{g} \mathrm{mg} \mathrm{C}{ }^{1}\right)$ and total body sterol concentrations $\left(r^{2}=0.61, P<0.001, t(27)=6.54\right)$.

In all animals, cholesterol was the predominant body sterol, representing between 69.6 (ergosterol treatment) and $92.5 \%$ (cholesterol treatment) of total body sterols (Fig. 4; Table A1, suppl. material). The only treatment in which cholesterol was less prominent in the animals $(42.0 \%)$ was the treatment in which the animals were reared on a diet supplemented with dihydrocholesterol. This treatment was characterized by a high percentage of the supplementary and presumably unprocessed dihydrocholesterol $(37.4 \%)$. In all other treatments, the
Table 1 Sterols used for supple mentation. Numbers in the $\Delta$ no tation indicate the position of double bonds in the sterol mole cule; C 24 alkylation refers to the presence of alkyl substituents at C24 in the side chain (cf. Fig. 5)

\begin{tabular}{llll}
\hline Trivial name & IUPAC name & Formula & $\Delta$ notation/C24 alkylation \\
\hline dihydrocholesterol & $5 \alpha$ cholestan $3 \beta$ ol & $\mathrm{C}_{27} \mathrm{H}_{48} \mathrm{O}$ & $\Delta^{0}$ \\
cholesterol & cholest 5 en $3 \beta$ ol & $\mathrm{C}_{27} \mathrm{H}_{46} \mathrm{O}$ & $\Delta^{5}$ \\
sitosterol & stigmast 5 en $3 \beta$ ol & $\mathrm{C}_{29} \mathrm{H}_{50} \mathrm{O}$ & $\Delta^{5} /$ ethyl \\
lathosterol & $5 \alpha$ cholest 7 en $3 \beta$ ol & $\mathrm{C}_{27} \mathrm{H}_{46} \mathrm{O}$ & $\Delta^{7}$ \\
7 dehydrocholesterol & cholesta 5,7 dien $3 \beta$ ol & $\mathrm{C}_{27} \mathrm{H}_{44} \mathrm{O}$ & $\Delta^{5,7}$ \\
brassicasterol & $(22 E)$ ergosta 5,22 dien $3 \beta$ ol & $\mathrm{C}_{28} \mathrm{H}_{46} \mathrm{O}$ & $\Delta^{5,22} /$ methyl \\
stigmasterol & $(22 E)$ stigmasta 5,22 dien $3 \beta$ ol & $\mathrm{C}_{29} \mathrm{H}_{48} \mathrm{O}$ & $\Delta^{5,22 / \text { ethyl }}$ \\
desmosterol & cholesta 5,24 dien $3 \beta$ ol & $\mathrm{C}_{27} \mathrm{H}_{44} \mathrm{O}$ & $\Delta^{5,24}$ \\
fucosterol & $(24 E)$ stigmasta $5,24(28)$ dien $3 \beta$ ol & $\mathrm{C}_{29} \mathrm{H}_{48} \mathrm{O}$ & $\Delta^{5,24(28) / \text { ethylidene }}$ \\
ergosterol & $(22 E)$ ergosta $5,7,22$ trien $3 \beta$ ol & $\mathrm{C}_{28} \mathrm{H}_{44} \mathrm{O}$ & $\Delta^{5,7,22 / \text { methyl }}$ \\
\hline
\end{tabular}


Fig. 2 Dietary threshold concentrations for sterol limited growth of Daphnia magna. Thresholds were defined as a growth rate reduction of $\mathrm{g}_{\infty}$ by $25 \%$. Data are presented as medians (horizontal lines within each box), quartiles (box), $10 \%$ and $90 \%$ percentiles (whiskers) and $95 \%$ confidence intervals (circles). Data labelled with the same letters are not significantly different $(P<0.05$; see methods for details)

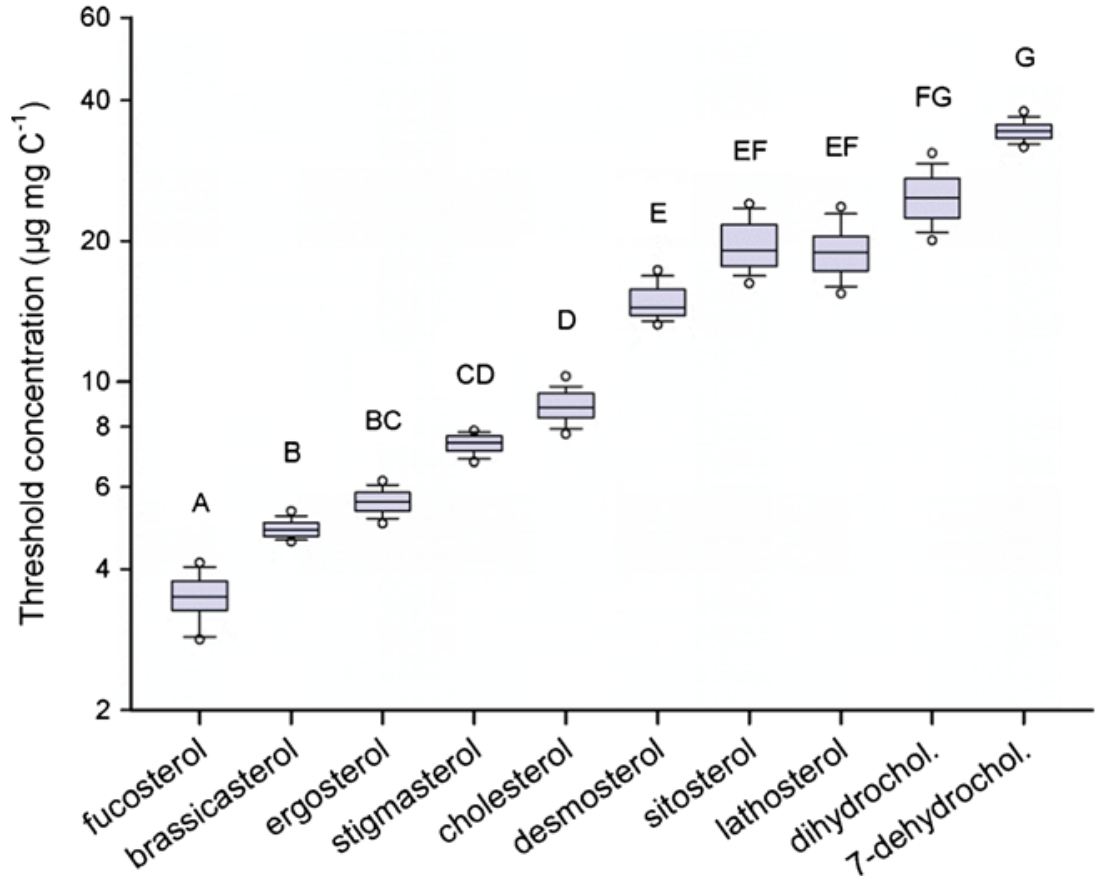

supplementary sterols also were detected in the animals, but in considerably lower concentrations, representing between 7.6 (ergosterol) and $15.1 \%$ (sitosterol) of total body sterols (Fig. 4). Besides cholesterol and the respective supplementary sterol, low to moderate concentrations of other sterols were detected in the animals (Fig. 4; Table A1, suppl. material). In animals reared on diets supplemented with stigmasterol, lathosterol, sitosterol, dihydrocholesterol, or ergosterol, respectively, these additional sterols represented $14.4-22.8 \%$ of total body sterols (in ascending order). In all other treatments (fucosterol, brassicasterol, cholesterol, desmosterol), less than $7.5 \%$ of total body sterols were represented by other sterols
(Fig. 4). Animals reared on the 7-dehydrocholesterol supplemented diet were the only animals in which only cholesterol and the supplemented sterol (i.e., 7-dehydrocholesterol) were detected (Table A1, suppl. material).

\section{Discussion}

The sterol-limited growth responses revealed significant differences among the supplementary sterols. Differences in the shape of the saturation growth curves resulted in different
Fig. 3 Sterol content of Daphnia magna (ng individual ${ }^{1}$ ) at the start of the experiment and after 5 $\mathrm{d}$ of feeding on either unsupplemented Synechococcus elongatus or $S$. elongatus supplemented with high concentrations ( $33 \mu \mathrm{g} \mathrm{mg} \mathrm{C}{ }^{1}$ ) of different sterols. Bars labelled with different letters are significantly different (Tukey's HSD, $P<0.05$ following ANOVA)

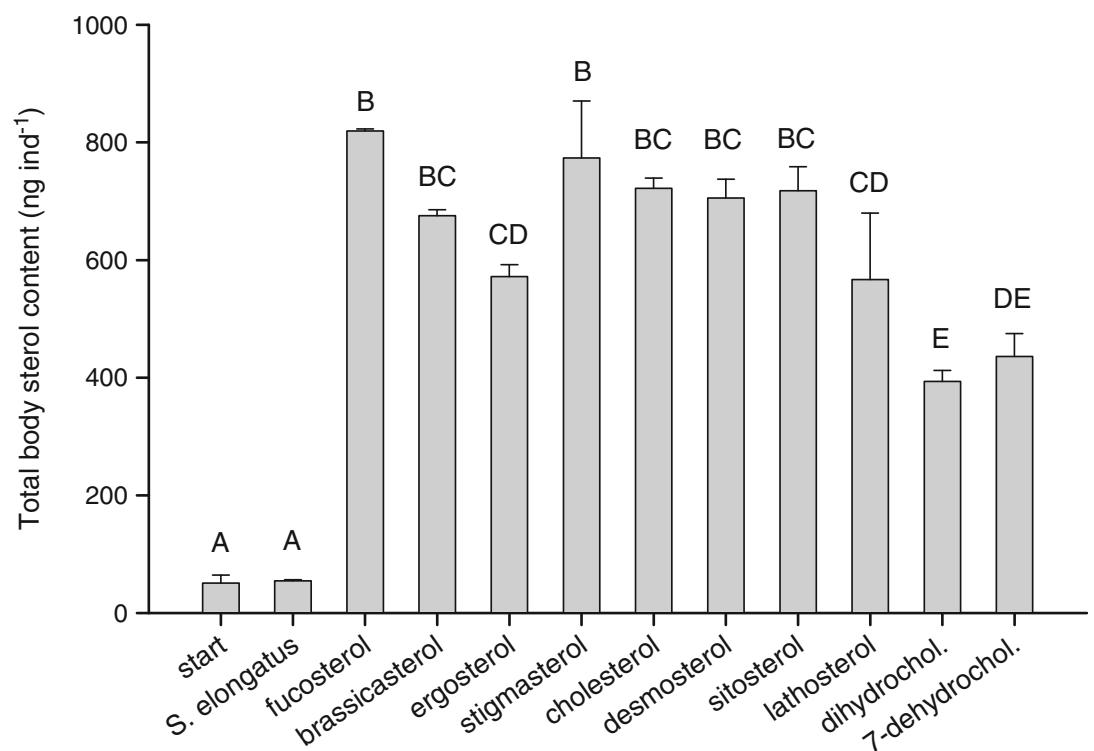


Fig. 4 Sterol composition of Daphnia magna. Animals were provided with high dietary concentrations ( $33 \mu \mathrm{g} \mathrm{mg} \mathrm{C}{ }^{1}$ ) of different sterols. Data show the relative contributions (\%) of cholesterol, the supplemented sterol, and the sum of other sterols detected in the animals to total body sterol concentrations. Error bars indicate standard deviations $(N=3)$

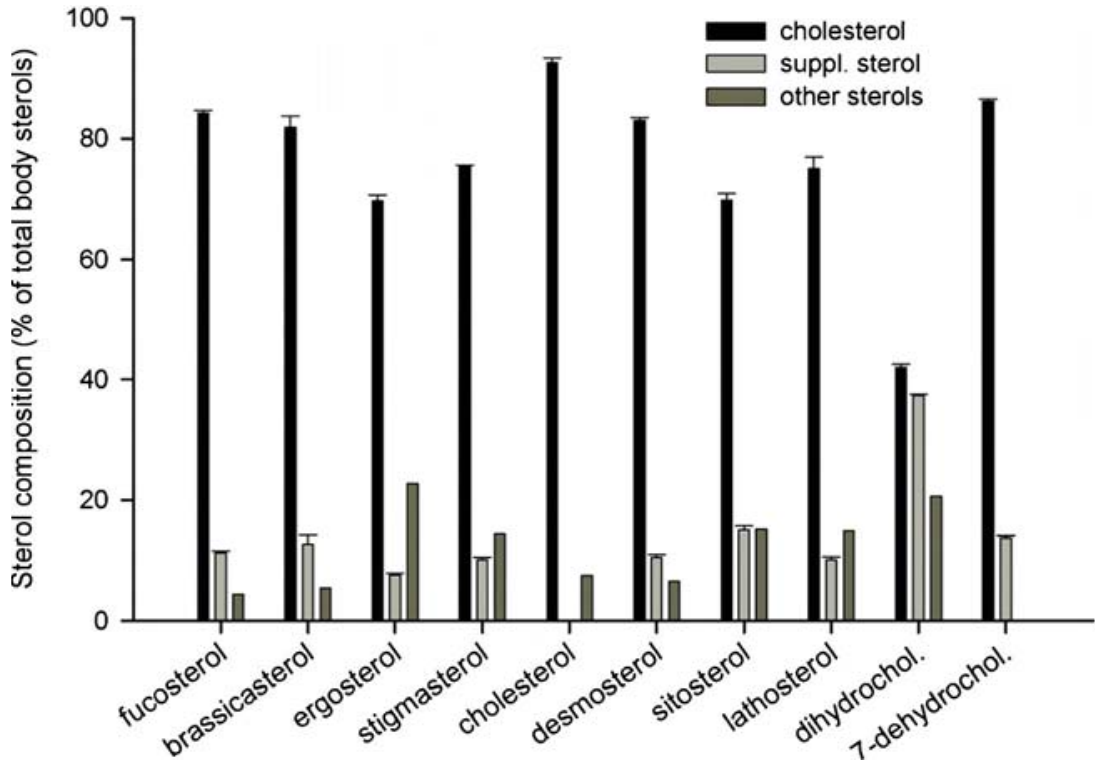

isomers), stigmasterol/poriferasterol (C-24 epimers), and/or brassicasterol/epibrassicasterol (C-24 epimers) have been found in some freshwater algae, such as Cryptomonas spp. and $N$. limnetica, and in the heterotrophic nanoflagellate Paraphysomonas sp., which are all high quality food sources for Daphnia (Bec et al. 2006; Martin-Creuzburg et al. 2005a, 2009; Piepho et al. 2010). Ergosterol is the predominant sterol in fungi, but significant concentrations also can be found in some freshwater green algae, such as Chlamydomonas spp. (Bec et al. 2006; Piepho et al. 2010; Thompson 1996). It should be noted, however, that high dietary concentrations of these sterols resulted in similar (fucosterol, ergosterol, stigmasterol) or only slightly higher (brassicasterol) growth rates than cholesterol. Thus, the differences in growth responses were due primarily to differences in the initial slope and the curvature of the growth curves, suggesting that qualitative differences among sterols are particularly important at low to moderate and thus potentially limiting dietary sterol concentrations.

In contrast to the sterols discussed above, supplementation with desmosterol, sitosterol, lathosterol, dihydrocholesterol, and 7-dehydrocholesterol revealed higher thresholds for sterol-limited growth than supplementation with cholesterol. The data suggest that these sterols are less efficient in supporting somatic growth than cholesterol and are required in higher concentrations than cholesterol to obtain the same growth response. At high dietary concentrations $(\geq 33 \mu \mathrm{g} \mathrm{mg}$ $\mathrm{C}^{1}$ ), however, supplementation with desmosterol and sitosterol revealed similar or even slightly higher growth rates than supplementation with cholesterol, resulting in an intersection of the growth curves. The growth response obtained by supplementation with 7-dehydrocholesterol revealed a similar trend. The curvature of the growth curve obtained with 7dehydrocholesterol was far less pronounced than the Significant concentrations of fucosterol/isofucosterol $(E / Z$ to take up and use certain phytosterols more efficiently than cholesterol, possibly because cholesterol is hardly represented in its algal diet. Appreciable amounts of cholesterol can be found only in certain algae, such as the eustigmatophyte Nannochloropsis limnetica (Martin-Creuzburg et al. 2009).

threshold concentrations at which sterol-saturated growth response obtained by experimentally increasing the availability of dietary cholesterol, the most relevant sterol in animal physiology, suggests that $8.9 \mu \mathrm{g} \mathrm{mg} \mathrm{C}^{1}$ of cholesterol is corroborates previous supplementation experiments, which suggested threshold concentrations for cholesterol between

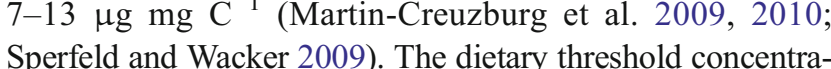
tions obtained by supplementing different sterols ranged between 3.5-34.3 $\mu \mathrm{g} \mathrm{mg} \mathrm{C}{ }^{1}$. Supplementation with fucosterol, brassicasterol, ergosterol, and potentially stigmasterol resulted same growth response as with cholesterol. This partially corroborates previous findings showing that ergosterol and stigmasterol are more efficient than cholesterol in supporting Elert 2004).

ol and the fact that cholesterol is the predominant sterol in animal tissues, one may expect that sterol assimilation mechanisms are optimized and acquire dietary cholesterol rather than phytosterols, which first have to be metabolized to cholesterol. However, our data suggest that D. magna is adapted in lower thresholds for sterol-limited growth than supplementation with cholesterol, suggesting that these sterols are more efficient in supporting somatic growth than cholesterol, and 
curvatures obtained with the other sterols, resulting in the highest threshold among all sterols tested. At high dietary concentration, however, growth rates obtained with 7dehydrocholesterol and cholesterol did not differ. These findings indicate that, for some sterols, poor quality can potentially be alleviated by increasing its dietary concentration.

Somatic growth rates also increased upon supplementation with lathosterol and dihydrocholesterol. However, the obtained growth curves were characterized by lower initial slopes and higher threshold concentrations than the growth curves obtained with cholesterol supplementation. At all dietary concentrations, even at the highest concentration, growth rates obtained with lathosterol and dihydrocholesterol supplementation were lower than those obtained with cholesterol supplementation, indicating that these sterols are less suitable for D. magna than cholesterol and the other phytosterols investigated here. Similarly, lathosterol and dihydrocholesterol have been shown to be less efficient in supporting somatic growth of D. galeata (Martin-Creuzburg and von Elert 2004).

It should be noted that the determination of threshold concentrations for sterol-limited growth is affected by the curvature of the growth curves. This might be relevant for growth curves with low initial slopes, such as the curves obtained with dihydrocholesterol or 7-dehydrocholesterol, which did not clearly converge to the maximum growth rates obtained by supplementation. To evaluate a potential overestimation due to the methods employed, the thresholds were recalculated. By assuming the highest sterol concentrations to be at 100 instead of $41 \mu \mathrm{g} \mathrm{mg} \mathrm{C}{ }^{1}$ and the corresponding growth rates to be equal to the ones obtained by supplementation with $41 \mu \mathrm{g} \mathrm{mg} \mathrm{C}{ }^{1}$. For 7-dehydrocholesterol, dihydrocholesterol, lathosterol, and sitosterol, the addition of these higher imaginary sterol concentrations resulted in slightly more pronounced curvatures of the growth curves and thus lower thresholds for sterol-limited growth (7dehydrocholesterol: 22.8 instead of $34.4 \mu \mathrm{g} \mathrm{mg} \mathrm{C}{ }^{1}$; dihydrocholesterol: 19.6 instead of $24.7 \mu \mathrm{g} \mathrm{mg} \mathrm{C}{ }^{1}$; sitosterol: 17.4 instead of $19.4 \mu \mathrm{g} \mathrm{mg} \mathrm{C}{ }^{1}$; lathosterol: 17.0 instead of $18.9 \mu \mathrm{g} \mathrm{mg} \mathrm{C}^{1}$ ). Hence, the thresholds reported for these sterols are potentially somewhat overestimated. For all other sterols, the shifts in thresholds obtained by adding the higher imaginary sterol concentration were negligible $(\leq 5 \%)$.

It remains to be established if Daphnia are capable of adjusting the intake of dietary sterols to cover their physiological demands. In a generalist grasshopper, it has been reported that the intake of unsuitable dietary sterols is regulated by post-ingestive feedback mechanisms and associative learning (Behmer et al. 1999a). In contrast to many terrestrial herbivores, daphnids are unselective filter feeders, and thus, presumably unable to equilibrate a dietary mismatch by compensatory feeding or selection of complementary food sources. However, it has been proposed that daphnids are capable of regulating the intake of elemental and essential biochemical nutrients via mechanisms operating post-ingestively in the gut (Anderson et al. 2005; Lukas and Wacker 2014).

To assess metabolic constraints potentially associated with the dietary sterol supply, we analyzed the sterol composition of animals reared on diets containing high concentrations (33 $\mu \mathrm{g} \mathrm{mg} \mathrm{C}^{1}$ ) of the different sterols. In animals reared on the cholesterol supplemented diet, $92.5 \%$ of total body sterols were represented by cholesterol and $7.5 \%$ by 7 dehydrocholesterol. In arthropods, 7-dehydrocholesterol $\left(\Delta^{5,7}\right)$ is an intermediate in the biosynthesis of the moltinducing ecdysteroids (Grieneisen 1994). In prothoracic glands of Bombyx mori, 7-dehydrocholesterol levels have been found to fluctuate with the molting cycle (Sakurai et al. 1986). Molt-related fluctuations may at least partially explain the varying concentrations of 7-dehydrocholesterol we found in D. magna (see Table A1, suppl. material). Animals feeding on the 7-dehydrocholesterol supplemented diet contained high amounts of cholesterol, indicating that D. magna is capable of converting dietary 7-dehydrocholesterol to cholesterol. A possible explanation for the restrained growth responses at low to moderate dietary concentrations of 7-dehydrocholesterol could be that the conversion of 7-dehydrocholesterol to cholesterol is rather inefficient and requires high substrate (i.e. 7dehydrocholesterol) concentrations. Daphnia magna provided with high dietary concentrations of 7-dehydrocholesterol achieved high growth rates and did not show any morphological abnormalities, suggesting that the incorporation of 7dehydrocholesterol did not interfere with ecdysteroid production.

A similar scenario to that described above for 7 dehydrocholesterol can be applied to the $\Delta^{7}$ sterol lathosterol. Dietary $\Delta^{7}$ sterols have been shown previously to constrain growth and/or development of several arthropod species (Behmer et al. 1999b; Klein Breteler et al. 1999; MartinCreuzburg and von Elert 2004). Moreover, it has been reported that C28 and C29 sterols containing double bonds at $\Delta^{5}$ or $\Delta^{5,7}$ are selectively removed from the diet during passage through the gut of a marine copepod, while $\Delta^{7}$ sterols are released unchanged as fecal lipids, suggesting that copepods possess mechanisms that avoid the assimilation of dietary $\Delta^{7}$ sterols (Prahl et al. 1984). We show here that lathosterol is assimilated by $D$. magna and used as cholesterol precursor. Besides lathosterol $\left(\Delta^{7}\right)$ and cholesterol $\left(\Delta^{5}\right)$, significant amounts of 7-dehydrocholesterol $\left(\Delta^{5,7}\right)$ were detected in animals reared on lathosterol supplemented diets, suggesting that cholesterol was synthesized from lathosterol via 7dehydrocholesterol $\left(\Delta^{7} \rightarrow \Delta^{5,7} \rightarrow \Delta^{5}\right.$; Fig. 5). The restrained growth responses observed upon lathosterol supplementation suggest that D. magna is inefficient in assimilating dietary lathosterol and/or in metabolizing lathosterol to cholesterol. These findings imply that crustacean zooplankton has limited capacities to use dietary $\Delta^{7}$ sterols. Sterols containing the $\Delta^{7}$ unsaturation predominate in certain green algae, such as 

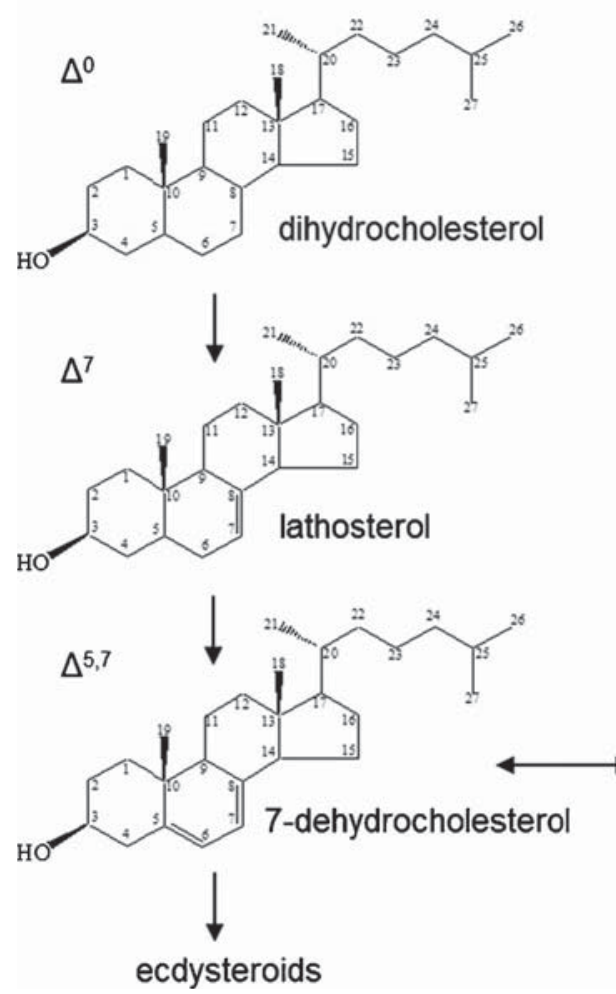

Fig. 5 Proposed routes of dietary sterol conversion in Daphnia magna. The different conversions may involve additional intermediate products not shown here. For instance, the conversion of $\mathrm{C} 28$ sterols, such as 22 dihydrobrassicasterol, to desmosterol presumably involves the formation of a $\Delta^{24,(28)}$ double bond to yield 24 methylenecholesterol and subse quently the formation of an epoxide prior to the loss of the C24 methyl group as it is found in various insects and decapod crustaceans (Cham berlain et al. 2004; Ikekawa et al. 1993; Kanazawa 2001; Svoboda 1999). Likewise, epoxidation prior to the actual dealkylation process is expected to be involved in the conversion of $\mathrm{C} 29$ sterols, such as stigmasterol or

Scenedesmus spp. (Klein Breteler et al. 1999; MartinCreuzburg et al. 2012; Piepho et al. 2010).

Similar to $\Delta^{7}$ sterols, ring-saturated sterols were found to pass quantitatively through the gut of copepods (Harvey et al. 1987). In our study, high proportions of dihydrocholesterol (37.4\%) were recorded in D. magna reared on the sitosterol, to desmosterol. This conversion presumably also involves the formation of fucosterol/isofucosterol ( $E / Z$ isomers), proposed intermedi ates in the conversion of sitosterol to desmosterol in insects and decapods (Ikekawa et al. 1993; Kanazawa 2001; Svoboda 1999). The carbon atoms are numbered according to IUPAC recommendations; capitals indicate the four rings (A, B, C, D) of the sterol nucleus. Numbers in the $\Delta$ notation indicate the position of double bonds in the sterol molecule; $\mathrm{C} 24$ alkylation refers to the presence of alkyl substituents at $\mathrm{C} 24$ in the side chain (cf. Table 1)

dihydrocholesterol supplemented diet. Combined with the low proportion of cholesterol $(42.1 \%$ ) detected in D. magna and the most restrained growth response among all sterols tested, this suggests limited capacities to assimilate and/or convert ring-saturated dietary sterols to cholesterol. Besides dihydrocholesterol $\left(\Delta^{0}\right)$ and cholesterol $\left(\Delta^{5}\right)$, comparatively 
large amounts of lathosterol $\left(\Delta^{7}\right)$ and 7-dehydrocholesterol $\left(\Delta^{5,7}\right)$ were detected in animals reared on the dihydrocholesterol supplemented diet, suggesting that cholesterol was synthesized from dihydrocholesterol via these intermediate products $\left(\Delta^{0} \rightarrow \Delta^{7} \rightarrow \Delta^{5,7} \rightarrow \Delta^{5}\right.$; Fig. 5). The finding that dihydrocholesterol and lathosterol are inefficient in supporting growth of $D$. magna corroborates previous results obtained with D. galeata (Martin-Creuzburg and von Elert 2004). In contrast to the previous study, however, in which only one sterol concentration was tested, we show here that the poor quality of dihydrocholesterol and lathosterol can be partially alleviated by increasing their dietary concentrations. Nevertheless, both studies show that dihydrocholesterol and lathosterol are less suitable for Daphnia than sterols containing the $\Delta^{5}$ unsaturation.

The sterol profiles of animals raised on diets supplemented with fucosterol or brassicasterol were characterized by high proportions of cholesterol. Supplementation with these sterols resulted in high somatic growth rates and low thresholds for sterol-limited growth, suggesting that they were efficiently assimilated and converted to cholesterol. Conversion of fucosterol $\left(\Delta^{5,24(28)} /\right.$ ethylidene $)$ and brassicasterol $\left(\Delta^{5,22} /\right.$ methyl) to cholesterol $\left(\Delta^{5}\right)$ requires dealkylation at C24 in the side chain and saturation of the $\mathrm{C} 24$ or $\mathrm{C} 22$ double bond, respectively. The detection of desmosterol in animals reared on the fucosterol and brassicasterol supplemented diets suggests that cholesterol is synthesized from these dietary precursors via desmosterol $\left(\Delta^{5,24}\right)$ (Fig. 5). In insects and decapod crustaceans, desmosterol has been identified as an intermediate in the metabolic pathway from C28 and C29 phytosterols to cholesterol (Ikekawa et al. 1993; Kanazawa 2001; Svoboda 1999), implying that similar pathways are active in D. magna.

Animals reared on diets supplemented with ergosterol or stigmasterol contained moderate concentrations of cholesterol and significant amounts of other non-supplementary sterols, yet showed growth responses similar to the animals raised on the cholesterol supplemented diet. The low thresholds for sterol-limited growth obtained with the ergosterol and stigmasterol supplemented diets indicate that these sterols are also highly effective in supporting somatic growth of D. magna. The sterol profile of animals reared on the ergosterol supplemented diet implies that cholesterol $\left(\Delta^{5}\right)$ was synthesized from ergosterol $\left(\Delta^{5,7,22} /\right.$ methyl $)$ via brassicasterol $\left(\Delta^{5,22} /\right.$ methyl) and 22-dihydrobrassicasterol ( $\Delta^{5} /$ methyl), requiring the consecutive saturation of two double bonds and the removal of the C24 methyl substituent (Fig. 5), a mechanism already proposed for decapod crustaceans (Kanazawa 2001). Animals reared on the stigmasterol ( $\Delta^{5,22} /$ ethyl) supplemented diet contained small amounts of sitosterol ( $\Delta^{5} /$ ethyl), suggesting that stigmasterol is first saturated at $\Delta^{22}$ to yield sitosterol and then dealkylated at $\mathrm{C} 24$ to yield cholesterol (Fig. 5). Isofucosterol and desmosterol, which both have been identified as intermediates in the conversion of sitosterol to cholesterol in insects and decapod crustaceans (Ikekawa et al. 1993; Kanazawa 2001), were undetectable in D. magna reared on the stigmasterol or sitosterol supplemented diets. We hypothesize, however, that the concentrations of isofucosterol and desmosterol in these animals were simply too low to be detectable and that the bioconversion of sitosterol to cholesterol in D. magna involves the same reactions as proposed for insects and decapod crustaceans (Fig. 5) (Grieneisen 1994; Ikekawa et al. 1993; Kanazawa 2001). Interestingly, supplementation with desmosterol or sitosterol resulted in significantly higher thresholds for sterol-limited growth than supplementation with cholesterol, indicating that at moderate dietary concentrations, desmosterol and sitosterol are less efficient in supporting somatic growth of D. magna. Considering that D. magna is capable of dealkylating dietary C28 and C29 phytosterols (e.g., sitosterol) to yield cholesterol and that desmosterol is an intermediate in this dealkylation process, this finding is surprising and thus should be subjected to further investigation.

As argued above, the different suitabilities of various dietary sterols to support animal growth may be due to differences in assimilation efficiencies or capacities to serve as cholesterol precursors. Moreover, the accumulation of unsuitable sterols within the body may interfere with physiological processes (e.g., ecdysteroid biosynthesis), and the incorporation of unsuitable sterols in cell membranes may have detrimental effects on various membrane properties (Haines 2001). In our study, the growth-promoting effects observed upon dietary sterol supplementation combined with the analysis of body sterols indicated that all supplemented sterols were to some extent assimilated. However, the concentrations of the supplemented sterols in D. magna and the obtained growth rates differed significantly among treatments, indicating strong differences in the efficiencies with which these dietary sterols were assimilated and further processed within the body. It also should be considered that dietary sterols may be modified by gut symbiotic microorganisms prior to assimilation (Clayton 1960; Nasir and Noda 2003). In contrast to other studies in which certain sterols were found to be highly detrimental for consumers (Behmer et al. 2011; Giner et al. 2003; Martin-Creuzburg and von Elert 2004), the supplementary sterols used in our study did not impose severe harmful or toxic effects on D. magna.

We show here that dietary phytosterols significantly differ in their potential to support somatic growth of D. magna, suggesting that the dietary sterol composition has to be considered in food quality studies to accurately assess a potential sterol limitation of crustacean zooplankton in the field.

Acknowledgments We thank P. Merkel for technical assistance in analyzing sterols. DMC was supported financially by the 'Young Scholar Fund' of the University of Konstanz, AW was supported by the German Research Foundation (DFG, WA 2,445/8 1). 


\section{References}

Anderson TR, Hessen DO, Elser JJ, Urabe J (2005) Metabolic stoichi ometry and the fate of excess carbon and nutrients in consumers. Am Nat 165:1 15

Basen T, Rothhaupt K O, Martin Creuzburg D (2012) Absence of sterols constrains food quality of cyanobacteria for an invasive freshwater bivalve. Oecologia 170:57 64

Bec A, Martin Creuzburg D, von Elert E (2006) Trophic upgrading of autotrophic picoplankton by the heterotrophic nanoflagellate Paraphysomonas sp. Limnol Oceanogr 51:1699 1707

Behmer ST, Elias DO, Bernays EA (1999a) Post ingestive feedbacks and associative learning regulate the intake of unsuitable sterols in a generalist grasshopper. J Exp Biol 202:739 748

Behmer ST, Elias DO, Grebenok RJ (1999b) Phytosterol metabolism and absorption in the generalist grasshopper, Schistocerca americana (Orthoptera : Acrididae). Arch Insect Biochem Physiol 42:13 25

Behmer ST, Grebenok RJ, Douglas AE (2011) Plant sterols and host plant suitability for a phloem feeding insect. Funct Ecol 25:484 491

Behmer ST, Nes WD (2003) Insect sterol nutrition and physiology: A global overview. Adv Insect Physiol 31:1 72

Bertalanffy LV (1957) Quantitative laws in metabolism and growth. Q Rev Biol 32:217 231

Bolker BM (2008) Ecological models and data in R. Princeton University Press, Princeton

Carvalho M et al (2010) Survival strategies of a sterol auxotroph. Development 137:3675 3685

Chamberlain PM, Bull ID, Black HIJ, Ineson P, Evershed RP (2004) Lipid content and carbon assimilation in Collembola: implications for the use of compound specific carbon isotope analysis in animal dietary studies. Oecologia 139:325 335

Clayton RB (1960) The role of intestinal symbionts in the sterol metabolism of Blattella germanica. J Biol Chem 235:3421 3425

Entchev EV, Kurzchalia TV (2005) Requirement of sterols in the life cycle of the nematode Caenorhabditis elegans. Semin Cell Dev Biol 16:175 182

Giner JL, Faraldos JA, Boyer GL (2003) Novel sterols of the toxic dinoflagellate Karenia brevis (Dinophyceae): A defensive function for unusual marine sterols? J Phycol 39:315 319

Goad LJ (1981) Sterol biosynthesis and metabolism in marine inverte brates. Pure Appl Chem 53:837 852

Grieneisen ML (1994) Recent advances in our knowledge of ecdysteroid biosynthesis in insects and crustaceans. Insect Biochem Molec Biol 24:115 132

Haines TH (2001) Do sterols reduce proton and sodium leaks through lipid bilayers? Prog Lipid Res 40:299 324

Harvey HR, Eglinton G, O'Hara SCM, Corner EDS (1987) Biotransformation and assimilation of dietary lipids by Calanus feeding on a dinoflagellate. Geochim Cosmochim Acta 51:3031 3040

Hassett RP (2004) Supplementation of a diatom diet with cholesterol can enhance copepod egg production rates. Limnol Oceanogr 49:488 494

Ikekawa N, Morisaki M, Fujimoto Y (1993) Sterol metabolism in insects: Dealkylation of phytosterol to cholesterol. Accounts Chem Res 26: 139146

Kanazawa A (2001) Sterols in marine invertebrates. Fisheries Sci 67: 9971007

Klein Breteler WCM, Schogt N, Baas M, Schouten S, Kraay GW (1999) Trophic upgrading of food quality by protozoans enhancing cope pod growth: role of essential lipids. Mar Biol 135:191 198

Lampert W (1991) The dynamics of Daphnia in a shallow lake. Verh Int Ver Limnol 24:795 798
Lukas M, Wacker A (2014) Daphnia's dilemma: adjustment of carbon budgets in the face of food and cholesterol limitation. J Exp Biol 217:1079 1086

Martin Creuzburg D, Bec A, von Elert E (2005a) Trophic upgrading of picocyanobacterial carbon by ciliates for nutrition of Daphnia magna. Aquat Microb Ecol 41:271 280

Martin Creuzburg D, Beck B, Freese HM (2011) Food quality of hetero trophic bacteria for Daphnia magna: evidence for a limitation by sterols. FEMS Microbiol Ecol 76:592 601

Martin Creuzburg D, Sperfeld E, Wacker A (2009) Colimitation of a freshwater herbivore by sterols and polyunsaturated fatty acids. Proc R Soc B 276:1805 1814

Martin Creuzburg D, von Elert E (2004) Impact of 10 dietary sterols on growth and reproduction of Daphnia galeata. J Chem Ecol 30:483 500

Martin Creuzburg D, von Elert E (2009) Ecological significance of sterols in aquatic food webs. In: Arts MT, Brett MT, Kainz MJ (eds) Lipids in aquatic ecosystems. Springer, New York, pp 43 64

Martin Creuzburg D, von Elert E, Hoffmann KH (2008) Nutritional constraints at the cyanobacteria Daphnia magna interface: The role of sterols. Limnol Oceanogr 53:456 468

Martin Creuzburg D, Wacker A, Basen T (2010) Interactions be tween limiting nutrients: Consequences for somatic and popu lation growth of Daphnia magna. Limnol Oceanogr 55:2597 2607

Martin Creuzburg D, Wacker A, von Elert E (2005b) Life history conse quences of sterol availability in the aquatic keystone species Daphnia. Oecologia 144:362 372

Martin Creuzburg D, Wacker A, Ziese C, Kainz MJ (2012) Dietary lipid quality affects temperature mediated reaction norms of a freshwater key herbivore. Oecologia 168:901 912

Moreau RA, Whitaker BD, Hicks KB (2002) Phytosterols, phytostanols, and their conjugates in foods: structural diversity, quantitative analysis, and health promoting uses. Prog Lipid Res $41: 457500$

Nasir H, Noda H (2003) Yeast like symbiotes as a sterol source in anobiid beetles (Coleoptera, Anobiidae): Possible metabolic pathways from fungal sterols to 7 dehydrocholesterol. Arch Insect Biochem Physiol $52: 175182$

Ohvo Rekilä H, Ramstedt B, Leppimäki P, Slotte JP (2002) Cholesterol interactions with phospholipids in membranes. Prog Lipid Res 41: 6697

Piepho HP (2004) An algorithm for a letter based representation of all pairwise comparisons. J Comput Graph Stat 13:456 466

Piepho M, Martin Creuzburg D, Wacker A (2010) Simultaneous effects of light intensity and phosphorus supply on the sterol content of phytoplankton. PLoS ONE 5:e15828

Porter JA, Young KE, Beachy PA (1996) Cholesterol modification of hedgehog signaling proteins in animal development. Science 274: 255259

Prahl FG, Eglinton G, Corner EDS, O'Hara SCM, Forsberg TEV (1984) Changes in plant lipids during passage through the gut of Calanus. J Mar Biol Assoc UK 64:317 334

Sakurai S, Yonemura N, Fujimoto Y, Hata F, Ikekawa N (1986) 7 dehydrosterols in prothoracic glands of the silkworm, Bombyx mori. Experientia 42:1034 1036

Sperfeld E, Martin Creuzburg D, Wacker A (2012) Multiple resource limitation theory applied to herbivorous consumers: Liebig's minimum rule vs. interactive co limitation. Ecol Lett 15:142 150

Sperfeld E, Wacker A (2009) Effects of temperature and dietary sterol availability on growth and cholesterol allocation of the aquatic keystone species Daphnia. J Exp Biol 212:3051 3059

Sperfeld E, Wacker A (2011) Temperature and cholesterol induced changes in eicosapentaenoic acid limitation of Daphnia magna 
determined by a promising method to estimate growth saturation thresholds. Limnol Oceanogr 56:1273 1284

Svoboda JA (1999) Variability of metabolism and function of sterols in insects. Crit Rev Biochem Mol 34:49 57

Thompson GA (1996) Lipids and membrane function in green algae. Biochim Biophys Acta 1302:17 45
Volkman JK (2003) Sterols in microorganisms. Appl Microbiol Biot 60: 495506

Wacker A, Martin Creuzburg D (2012) Biochemical nutrient re quirements of the rotifer Brachionus calyciflorus: co limitation by sterols and amino acids. Funct Ecol 26:1135 1143 